\title{
Summary of ICF 11
}

\author{
S. NAKAGAWA ${ }^{\text {a)* }}$ and T. TANIYAMA ${ }^{\text {b)* }}$ \\ a) Dept. of Physical Electronics, Tokyo Institute of Technology, 2-12-1 Ookayama, Meguro-ku, Tokyo 152-8552, Japan \\ b) Materials \& Structures Laboratory, Tokyo Institute of Technology, 4259 Nagatsuta, Midori-ku, Yokohama 226-8503, Japan
}

Abstract

The 11th International Conference on Ferrites, ICF11, was successfully held on April 15-19, 2013, at the Okinawa Convention Center, Okinawa, Japan organized by The Japan Society of Powder and Powder Metallurgy. ICF 11 covered a broad range of topics related to the science and technology of ferrites and related materials.

\section{INTRODUCTION}

The 11th International Conference on Ferrites, ICF 11, was held at the Okinawa Convention Center, Okinawa, Japan organized by The Japan Society of Powder and Powder Metallurgy on April 15-19, 2013. The ICF 11 was initially scheduled to be held in Nara, Japan, in 2011 but was postponed once due to the Great East Japan Earthquake. The organizing committee rescheduled the conference for April 2013. Earlier ICF's were held in Kyoto (1970, 1980, and 2000), Paris (1976), San Francisco (1984 and 2004), Mumbai (former Bombay) (1989), Tokyo (1992), Bordeaux (1996), and Chengdu (2008). The ICFs have been providing a forum for exchanging information on the fast-developing science and technology of ferrites and related materials that promise to be important for the electronic industry. Presently, the exciting and central subject of ferrites is spreading beyond the traditional boundaries of physics and chemistry, electronics and devices, and biology and medical sciences. To meet the pressing need to hold a conference on the key issues not only of ferrites but also of other advanced materials, ICF 11 covered a broad range of topics related to the science and technology of such materials.

\section{SPONSORSHIP}

Organized by

The Japan Society of Powder and Powder Metallurgy

Supported by

The Science Council of Japan

The Ministry of Education, Culture, Sports, Science and Technology, Japan

Commemorative Organization for the Japan World Exposition ('70), Japan

The Murata Science Foundation, Japan

Cooperated by

The Magnetic Society of Japan

The Physical Society of Japan

The Japan Society of Applied Physics

The Institute of Electrical Engineers of Japan

IEEE Japan Council, Magnetics Society, Japan Chapter

The Electrochemical Society of Japan

The Institute of Electronics Information and Communication Engineers

The Chemical Society of Japan

The Japan Institute of Metals

The Ceramics Society of Japan

Technical Sponsoring by

American Ceramics Society

\footnotetext{
* Co-chairmen of Program Committee, ICF 11
} 


\section{TECHNICAL PROGRAMS}

The ICF 11 included 3 plenary talks, 4 symposia, 16 oral sessions and 2 poster sessions. During the conference period, 260 papers, consists of 109 oral presentations and 151 poster presentations were performed. Time table of the ICF 11 is indicated as Table.1. Some snapshots during the conference are indicated in Fig.1.

\section{Plenary Talks}

Ramamoorthy Ramesh, University of California, Berkeley, USA

"Electric Field Control of Magnetism Using Multiferroics"

Hideo Hosono, Tokyo Institute of Technology, Japan

"Iron-Based Superconductors: Current Status"

John Paul Attfield, University of Edinburgh, UK

"The Verway Phase of Magnetite - a Long-Running Mystery in Ferrites"

\section{Symposia}

- Biomedical applications of magnetic particles and structures

- Multiferroic materials: physics and applications

- Hard magnetic materials and their applications

- Environmental preservation by using magnetic particles and superconducting magnets

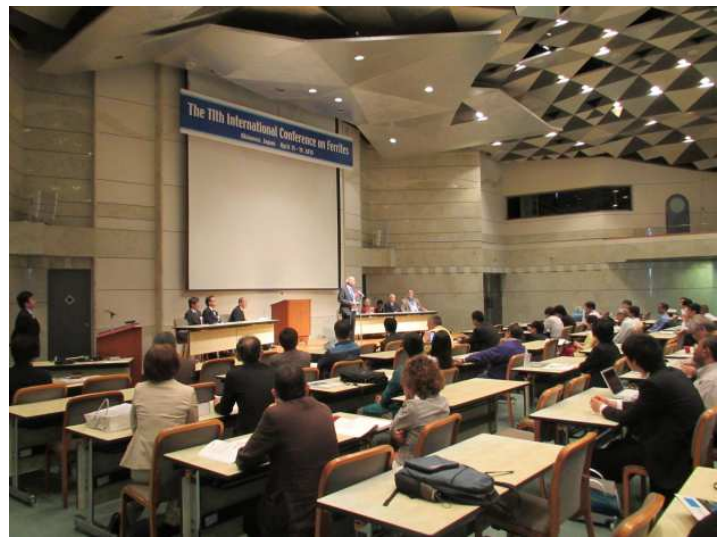

(a) Opening Session

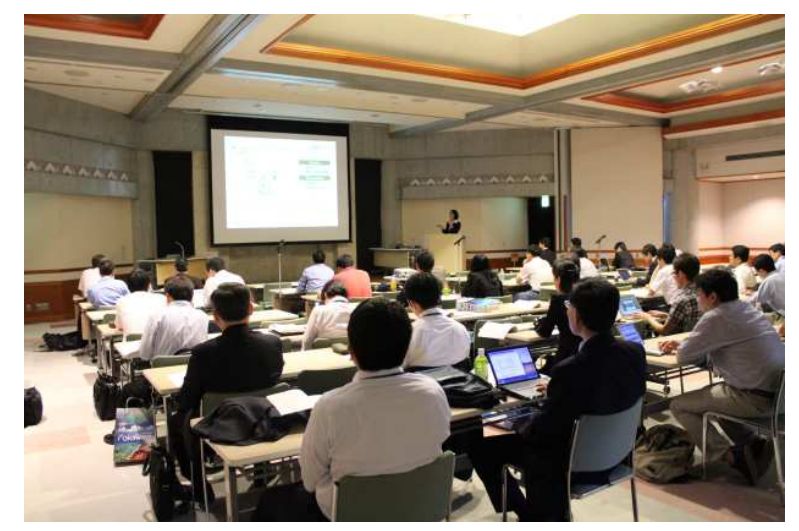

(c) Oral Session

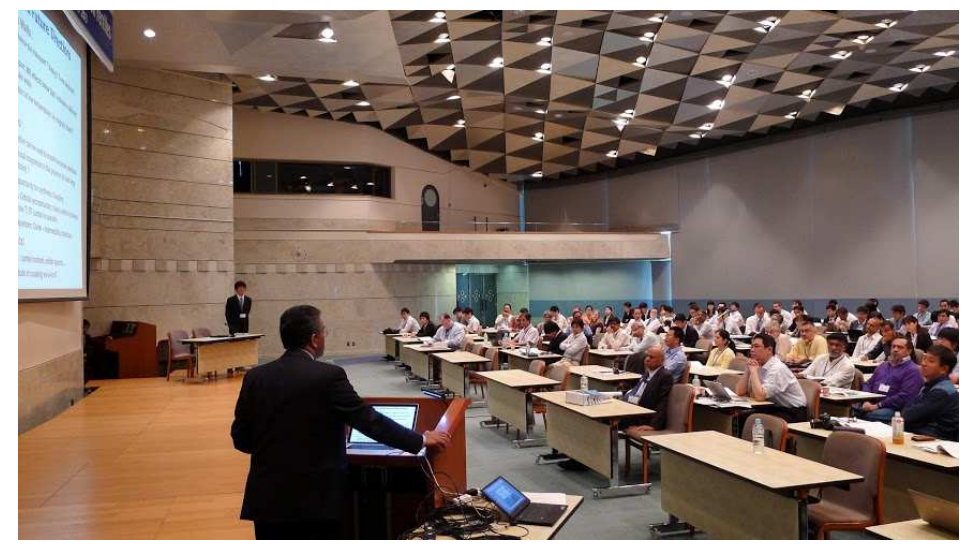

(b) Plenary Talk

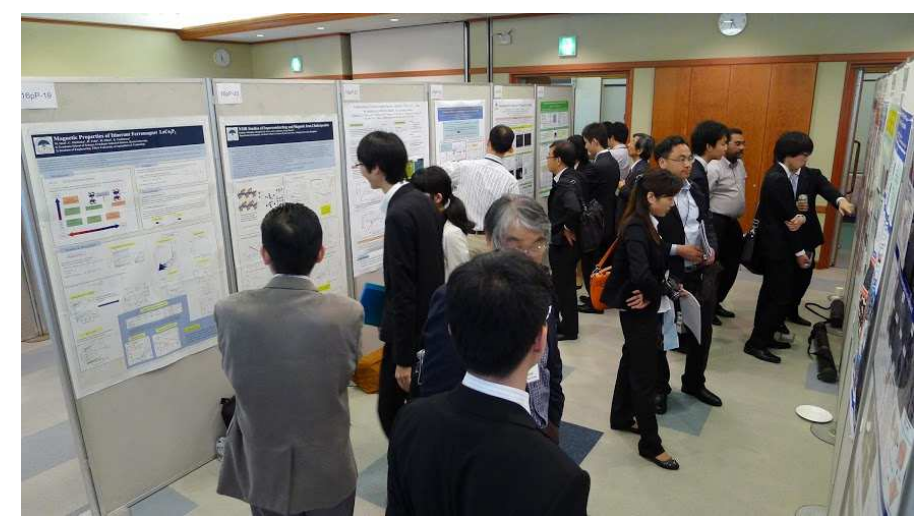

(d) Poster Session

Fig. 1 Snapshots of ICF 11 
Table 1 Time Table of ICF 11

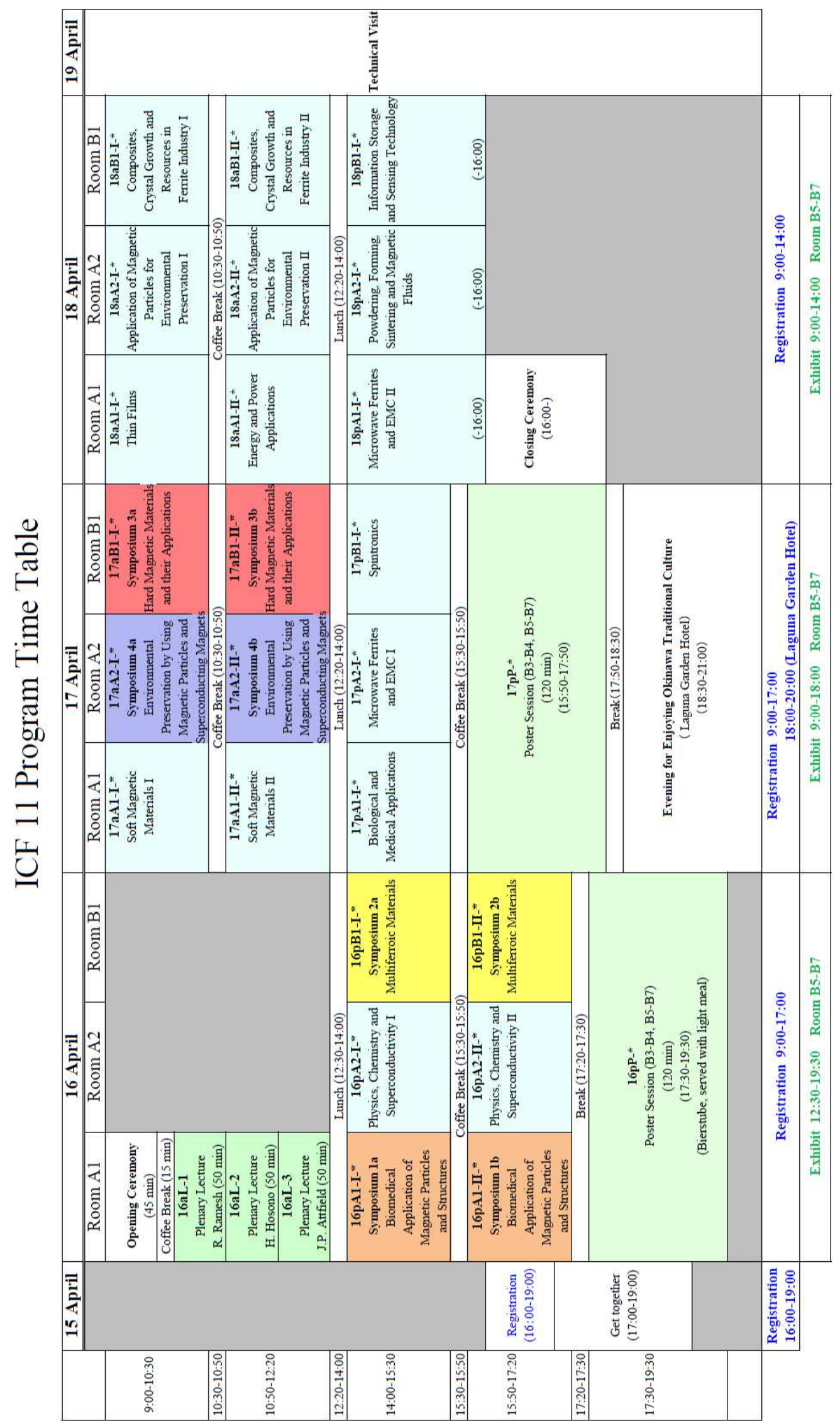




\section{AWARDS OF ICF 11}

\section{Takeshi Takei Award}

Takeshi Takei Award was given to Prof. C. M. Srivastava of the Indian Institute of Bombay - the father of ferrites in India - for his contribution to ferrites in India and support for the ICF conferences.

\section{Young Researcher's Award}

This award was given to outstanding papers presented at ICF 11, in either oral or poster format, by researchers not older than 35 years. Award winners are as follows.

Ilsiyar Ildusovna Nigmatullina (Kazan Federal University)

"Measurement of size of ferromagnetic clusters in $\mathrm{Tm}_{0.65} \mathrm{Sr}_{0.35} \mathrm{Mn}_{1-x} \mathrm{Fe}_{x} \mathrm{O}_{3}$ according to the ESR spectra" $[16 \mathrm{pP}-11]$

Tomohiro Nozaki (Tohoku University)

"Orientation Control of $\mathrm{Cr}_{2} \mathrm{O}_{3}$ Thin Film Crystallized by Post-annealing" [16pP-30]

Juji Kato (FDK Corporation)

"Magnetic Properties of Bi and Si Oxides-doped M-Type Barium Ferrite" [17pP-19]

Naonori Sakamoto (Shizuoka University)

"Synthesis of bio-compatible ( $\mathrm{La}, \mathrm{Sr}) \mathrm{MnO}_{3}-\mathrm{HAp}$ hybrid particles and their hyperthermia properties" [17pP-67]

Siti Aisyah Binti Shamsudin (Kyoto University)

"Introduce the Safe Capsule for CdS Quantum Dots as Bio-labeling Device" [17pP-69]

Hajime Hojo (Tokyo Institute of Technology)

"Crystal Structure and Electrical Properties of $(1-x) \mathrm{BiFeO}_{3}-x \mathrm{BiCoO}_{3}$ Thin Films Prepared by Pulsed Laser Deposition" [18aA1-I-5]

Tomohiko Niizeki (University of Tsukuba)

"Large Perpendicular Magnetic Anisotropy in Fe-rich Cobalt Ferrite Epitaxial Thin Film Grown by a Reactive Magnetron Sputtering Technique" [18pB1-I-5]

\section{New Product \& Novel Technology Award}

This award was given for exceptional "new products" and "novel technologies" related to ferrites and related materials presented at ICF 11 in oral/poster sessions or technical exhibit. Award winners are as follows.

Y. Namiki (The Jikei University School of Medicine)

"A Rapid and Reliable Elimination System for Radioactive Cesium using Magnetic Nanocomposites"

A. Sandhu, T. Takamura (Toyohashi University of Technology)

"MISA-Diagnostic System Medical Diagnostic Protocol based on Magnetically Induced Self-Assembly of Superparamagnetic Beads"

M. Inoue, H. Takagi, P. B. Lim (Toyohashi University of Technology)

"Novel 3D Display using Garnet-based Magnetophotonic Crystals with Large-Number Magneto-Optic Nano-Pixel"

Y. Ichiyanagi, H. Takeuchi, D. Shigeoka (Yokohama National University)

"Optimization of Ferrite Nanoparticles as an Agent for Hyperthermia Treatment"

K. Hagita, H. Kobayashi, F. Sato, Y. Yazaki, M. Sonehara, T. Sato, T. Watanabe, N. Matsushita, T. Fujii, H. Shimizu, K. Kobayashi (Shinshu University, Tokyo Institute of Technology, SHINKO Electric Industries)

"Magnetic Properties of Spin-sprayed Ferrite Film Deposited on Glass/Epoxy Build-up Layer in Organic Interposer Substrate"

E. Wada, Y. Shirahata, M. Itoh, M. Yamaguchi, T. Taniyama (Tokyo Institute of Technology, Nagoya University)

"Electron Spin Transmission due to Spin-Filtering Effect in $\mathrm{Fe}_{3} \mathrm{O}_{4}$-based Spin-LED"

M. Naoe, N. Kobayashi, S. Ohnuma, T. Iwasa, K. Ishida, H. Masumoto, K. I. Arai (Research Institute for Electromagnetic Materials, Tohoku University) 
"Nano-Granular Soft Magnetic Thin Films Having Both Ultra-high Resistivity and Magnetization More Than Ferrite"

S. Takeda, S. Motomura, T. Hotch, H. Suzuki (Magnontech, Ltd., KEYCOM Corp.)

"High Frequency Magnetic Measurement System from $100 \mathrm{kHz}$ to $10 \mathrm{GHz}$ "

T. Hasegawa, R. Nakajima, S. Ichiguchi, T. Wada, M. Koshino, T. Okada (Murata Manufacturing Co., Ltd.)

“A Miniaturized Low Loss Lumped Element Isolator Operating in Weak Magnetic Field below Ferromagnetic Resonance"

T. Yasuoka, S. Matsuzawa, T. Chiba, K. Murai, Y. Yamauchi, K. Harada (NEC TOKIN Corporation)

"High Impedance MnZn Ferrite "S15H" and Common Mode Choke Coil "SCR" ",

T. Ishibashi, K. Sato, M. Nagai, A. Mizusawa, T. Kosaka, T. Nagane, M. Kawahara (Nagaoka University of Technology, Japan Science and Technology Agency, Tokyo University Agriculture and Technology, Kojundo Chemical Laboratory Co., Ltd.)

"MOD Solutions for Magnetic Garnet Films"

Y. Manabe, T. Mizokami, T. Nishimoto (Kobe Steel, Ltd., Shinko Techno Engineering Co., Ltd.)

"DIP (Dry Integrated Press) Equipment"

H. Ogawa, A. Tanaka, H. Matsuura, K. Tanaka, H. Kishi, K. Kawano (TAIYO YUDEN Co., Ltd.)

"Power Inductor using New Metal Composite Material MCOIL(TM)"

\section{Yamazaki Yohtaro Memorial Student Award}

This award was given for outstanding papers presented by students at ICF 11 in either oral or poster formats. The Award is made in commemoration of the late Dr. Yamazaki Yohtaro, Emeritus Professor of Tokyo Institute of Technology, who has made outstanding achievements in ferrite technology and made great contributions to ICF 6 as Social Arrangements, and ICF 8 as Program Chairman, and for a limited period as Secretary of ICF 11. Dr. Yohtaro Yamazaki passed away in February 2012 at 66 years old. Award winners are as follows.

Masaki Imai (Kyoto University)

Magnetic Properties of Itinerant Ferromagnet $\mathrm{LaCo}_{2} \mathrm{P}_{2}[16 \mathrm{pP}-19]$

Aiko Kawahito (Hokkaido University)

Fabrication of Fe Nanowires by Thermal CVD Method [16pP-23]

JeongSoo Hong (Tokyo Institute of Technology)

The Crystallographic Property of $\mathrm{Fe}_{3} \mathrm{O}_{4}$ layer Deposited Successuvely on ZnO layer by Spin-Spray Method [16pP-39]

George Kogias (Centre for Research and Technology-Hellas)

New MnZn Ferrites with High Saturation Flux Density [17aA1-I-4]

Marie Yoshikiyo (The University of Tokyo)

Synthesis, Magnetic Properties, and Theoretical Studies of e-Fe2O3 [17pP-48]

Hiroki Morita (Tokyo University of Science)

Design and Fabrication of High-Pressure Reaction Vessel for High-throughput Magnetic Materials Exploration [17pP-58]

Ryo Sakuma (Okayama University)

Characterization of Microtubule Material Consisting of Iron Oxide Nanoparticles Produced by Bacteria [17pP-62]

Shofu Matsuda (Waseda University)

Induction of Cell Death and Uptake in Mesothelioma Cells by Magnetite Nanoparticles [17pP-70]

Ruzhi Zhang (Tokyo Institute of Technology)

Magnetic and optical nanohybrids composed of FePt/Au nanoshell [17pP-75]

Takao Kashima (Tokyo Institute of Technology)

Fabrication of $\mathrm{FeO}$ films with (100) orientation for underlayer of $\mathrm{CoFe}_{2} \mathrm{O}_{4}$ thin film [18aB1-II-3] 


\title{
V. EXHIBITS
}

Exhibits were performed by the following exhibitors throughout the conference period. (Alphabetical order)

\section{EXHIBITORS of ICF 11}

\author{
BASF Japan Ltd. \\ EPSON ATMIX CORPORATION \\ FDK CORPORATION \\ FUJITSU LIMITED \\ KEYCOM Corporation \\ Kobe Steel, Ltd \\ KOJUNDO CHEMICAL LABORATORY CO., LTD. \\ MITSUWA FRONTECH CORP. \\ NEC TOKIN Corporation \\ Riedhammer $\mathrm{GmbH}$. \\ SINTOKOGIO, LTD. \\ TAIYO YUDEN CO., LTD. \\ TDK Corporation \\ UNICO LTD
}

\section{PARTICIPANTS OF ICF 11}

There were 286 participants from 19 countries. The per country list is indicated in Table 2.

Table 2 Per country list for participants in ICF 11.

$\begin{array}{lllr}\text { Austria } & 2 & \text { Japan } & 219 \\ \text { China } & 6 & \text { Korea } & 13 \\ \text { Egypt } & 1 & \text { Latvia } & 1 \\ \text { Finland } & 1 & \text { Malaysia } & 1 \\ \text { France } & 3 & \text { Mexico } & 1 \\ \text { Germany } & 8 & \text { Poland } & 1 \\ \text { HongKong } & 2 & \text { Taiwan } & 5 \\ \text { India } & 6 & \text { U.K. } & 5 \\ \text { Indonesia } & 1 & \text { U.S.A. } & 9 \\ \text { Italy } & 1 & & 286\end{array}$

\section{TECHNICAL VISITS OF ICF 11}

Technical Visits, the final event of ICF 11, to Okinawa Churaumi Aquarium took place on April 19. The aquarium is one of the most attractive facilities of the Okinawa Ocean Expo Park located at the waterfront of north part of Okinawa Main Island. About 70 participants of ICF 11 take took part in this technical visit. The special scientific lecture "Research activity in Okinawa Churaumi Aquarium - Contribution to the Science of Conservation Biology" was given by Dr. Keiichi Sato of Okinawa Churaumi Aquarium. More detailed information about the technical visit is given in "Social Activities (Get-Together, Banquet, Technical Visit) Held in ICF 11". 


\section{COMMITTEE MEMBERS}

\section{The ICF International Committee Members}

Chairman:

M. Sugimoto (Japan)

Honorary Members:

V. Cagan (France), A. Goldman (U.S.A.), C.M. Srivastava (India)

Members:

Y. Bando (Japan), G. Bottoni (Italy), V.A.M. Brabers (The Netherlands), H. Chen (China), M. Guyot (France),

F. Haberey (Germany), V.G. Harris (U.S.A.), B.A. Kalinikos (Russia), A.H. Morrish (Canada), M. Naoe (Japan), H. Szymczak (Poland), G. Thomas (U.S.A.), V.D. Volonkov (Russia), I.P. Wilkinson (U.K.)

\section{Executive Committee Members}

Chairman

Mitsuo Sugimoto (Chairman of ICFIC)

Vice Chairman

Masanori Abe (Prof. Emeritus at Tokyo Institute of Technology)

Secretary

Nobuhiro Matsushita (Tokyo Institute of Technology)

Program

Shigeki Nakagawa (Tokyo Institute of Technology)

Tomoyasu Taniyama (Tokyo Institute of Technology)

Proceedings

Satoshi Sugimoto (Tohoku University)

Takashi Nakagawa (Osaka University)

Financial

Hitoshi Taguchi (TDK Corporation)

Naoyoshi Sato (TDK Corporation)

Contribution

Nobuyuki Hiratsuka (Saitama University)

Yoshitaka Kitamoto (Tokyo Institute of Technology)

Local Arrangement

(Kansai) Kazuyoshi Yoshimura (Kyoto University)

Nobuyuki Hosoito (Nara Institute of Science and Technology)

Local Arrangement

(Okinawa) Eiji Kaneko (Ryukyu University)

Kenichi Yamamoto (Ryukyu University)

Social Arrangement

Shigeru Ito (Tokyo University of Science)

Yoshio Matsuo (FDK Corporation)

Exhibit

Setsuo Yamamoto (Yamaguchi University)

Shigeyoshi Yoshida (NEC Tokin Corporation)

Hiroshi Kishi (Taiyo Yuden Corporation) 


\section{Program Committee Members}

Co-chair: Shigeki Nakagawa (Tokyo Institute of Technology)

Co-chair: Tomoyasu Taniyama (Tokyo Institute of Technology)

Masanori Abe (Tokyo Institute of Technology)

Manabu Gomi (Nagoya Institute of Technology)

Koichi Haneda (Ishinomaki Senshu University)

Nobuyuki Hiratsuka (Saitama University)

Mitsuteru Inoue (Toyohashi University of Technology)

Eiji Kita (University of Tsukuba)

Osamu Kitakami (Tohoku University)

Kimihide Matsuyama (Kyushu University)

Hiroaki Muraoka (Tohoku University)

Tatsuya Nakamura (University of Hyogo)

Tomoyuki Ogawa (Tohoku University)

Shin-ichi Ohkoshi (Tokyo University)

Kenji Ohmori (The Japan Association of Bonded Magnetic Materials)

Hiroshi Sakuma (Utsunomiya University)

Takao Sasagawa (Tokyo Institute of Technology)

Naoyoshi Sato (TDK Corporation)

Satoshi Sugimoto (Tohoku University)

Migaku Takahashi (Tohoku University)

Yasushi Takemura (Yokohama National University)

Yutaka Tamaura (Tokyo Institute of Technology)

Masahiro Yamaguchi (Tohoku University)

Hiroshi Yamamoto (Meiji University)

Yohtaro Yamazaki (Tokyo Institute of Technology)

Hideto Yanagihara (Tsukuba University)

Shigeyoshi Yoshida (NEC TOKIN Corporation)

\section{Program Committee Working Group}

Chair: Shigeki Nakagawa (Tokyo Institute of Technology)

Masanori Abe (Tokyo Institute of Technology)

Takuya Aoki (TDK Corporation)

Shigeo Fujii (Hitachi, Ltd.)

Hideaki Fukuzawa (TOSHIBA CORPORATION)

Takashi Hasegawa (Murata Manufacturing Co., Ltd.)

Satoshi Hirosawa (Hitachi Metals, Ltd.)

Kenji Kawano (TAIYO YUDEN CO., LTD.)

Koichi Kondo (NEC TOKIN Corporation)

Yoshio Matsuo (FDK CORPORATION)

Nobuhiro Matsushita (Tokyo Institute of Technology)

Takashi Nakagawa (Osaka University)

Masayuki Naoe (Research Institute for Electromagnetic Materals)

Ken Ohashi (Shin-Etsu Chemical Co., Ltd.) 
Yukiko Okazaki (JFE Chemical Corporation)

Kiyoto Ono (FDK CORPORATION)

Tomoyasu Taniyama (Tokyo Institute of Technology)

Yohtaro Yamazaki (Tokyo Institute of Technology) 